\section{Goldmann tonometer calibration: a national survey}

\begin{abstract}
Introduction Recent studies suggest that Goldmann tonometers can rapidly develop calibration errors (CEs) in clinical use and routine checks are necessary to ensure

tonometers being used in clinical practice. We suggest that every eye unit should have a protocol, which clearly identifies individuals responsible for checking for CEs at least on a monthly basis.
\end{abstract} accuracy.

Purpose To determine current practice regarding $\mathrm{CE}$ checks in the United Kingdom and assess the views of senior nursing staff in charge of running ophthalmology outpatient clinics as to whom they feel to be responsible for CE checks.

Methods Every ophthalmology unit with training recognition in England, Northern Ireland, Scotland, and Wales was contacted. Senior nurses responded to a structured telephone questionnaire regarding local tonometer calibration practice and their views regarding who is responsible for $\mathrm{CE}$ checks. A total of 155 eye units were identified and contacted. The response rate was $\mathbf{1 0 0 \%}$.

Results CEs were checked for daily in 8 units (5.2\%), weekly in 20 units (12.9\%), fortnightly in 1 unit $(0.6 \%)$, monthly in 12 units $(7.7 \%)$, trimonthly in 5 units (3.2\%), biannually in 27 units (17.4\%), and annually in 21 units (13.5\%). CEs were either never checked or checked in a very random manner (no identifiable pattern) in 61 units $(39.4 \%)$. Sixty-three $(40.6 \%)$ of the respondents felt $\mathrm{CE}$ checks were a departmental responsibility, 48 (31.0\%) felt it to be the doctor's responsibility, and $44(28.4 \%)$ felt $\mathrm{CE}$ checks should be performed by the nursing staff.

Conclusions Our national survey suggests that very few units check their tonometers for CEs at intervals which ensure their accuracy. Our previous survey of doctors suggests that they believe nurses should check for $C E$, whereas the nursing staff believe $C E$ checks are not their responsibility. This lack of communication between health-care professionals may lead to inaccurate
Eye (2009) 23, 413-415; doi:10.1038/sj.eye.6703010; published online 26 October 2007

Keywords: Goldmann applanation tonometer; calibration errors; practice in UK

\section{Introduction}

Despite being invented more than fifty years ago, Goldmann applanation tonometry remains the gold standard for the measurement of intraocular pressure (IOP). Haag-Streit (Koeniz-Berne, Switzerland) recommends calibration errors (CEs) of its Goldmann tonometers should remain within $\pm 0.5 \mathrm{mmHg}$, and that any tonometer found to be outside this range should be removed from clinical practice and returned to the manufacturer for recalibration. ${ }^{1}$ However, they provide no guidelines or protocol as to how often the $\mathrm{CE}$ checks should be made. ${ }^{1}$ A recent publication suggests that annual checking is normal practice. However, this is deemed to be insufficient to ensure tonometer accuracy. ${ }^{2,3}$ The importance of timely CE checks has been emphasised by a recent paper, which suggested that tonometers lose their accuracy during routine clinical practice and may develop CE, which frequently over-estimate IOP. ${ }^{2}$ A further issue which has not been addressed is whose responsibility it should be to ensure that tonometer calibration does take place. Doctors believe that $\mathrm{CE}$ checks are not their responsibility, and only a minority perform these checks on the tonometers they use. As each tonometer may be used by different doctors on a daily basis depending on the slit-lamp or clinic room used, this may place

N Kumar and RJ Hillier

Ophthalmology Department, Aintree University Hospitals NHS Foundation Trust, Walton Hospital, Rice Lane, Liverpool, UK

Correspondence: N Kumar, $\mathrm{SpR}$ in Ophthalmology, 185 Waterloo Warehouse, Waterloo Road, Liverpool L3 OBQ, UK Tel: + 07951577708 . E-mail: nishant6377@ gmail.com

Received: 27 May 2007 Accepted in revised form: 4 September 2007 Published online: 26 October 2007

This survey was presented as a poster at the Royal College of Ophthalmologists Annual Congress, May 2006. 
tonometer accuracy at risk. ${ }^{3}$ This study evaluates the current practice regarding $\mathrm{CE}$ checks in the United Kingdom and assesses the views of senior nursing staff in charge of outpatient clinics as to whom they believe to be responsible for $\mathrm{CE}$ checks in their department.

\section{Materials and methods}

Every ophthalmology unit with training recognition in England, Ireland, Scotland, and Wales was identified from the Royal College of Ophthalmologists' Directory of Training Posts in Ophthalmology 2005-2006. At each institution, a senior nurse familiar with the day-to-day running of the outpatient ophthalmology clinic responded to a structured telephone questionnaire regarding the Goldmann tonometer CE checks. A followup telephone call was made when the respondent needed to check the details we required or was unavailable to participate due to clinical time constraints.

Respondents were asked how often all Goldmann tonometers in clinical practice were checked for $\mathrm{CE}$, and whom they felt to be responsible for checking tonometers for CE (doctor/nurse/departmental responsibility).

\section{Results}

A total of 155 eye units were identified in the UK and contacted. A 100\% response rate was achieved. All responding units were using Goldmann applanation tonometers in routine clinical practice.

CEs were checked for daily in 8 units $(5.2 \%)$, weekly in 20 units $(12.9 \%)$, fortnightly in 1 unit $(0.6 \%)$, monthly in 12 units $(7.7 \%)$, trimonthly in 5 units $(3.2 \%)$, biannually in 27 units (17.4\%), and annually in 21 units (13.5\%). CEs were either never checked or checked in a very random manner (no identifiable pattern) in 61 units (39.4\%). Units where the CEs was checked 'occasionally', 'never', 'if required', or 'in no particular time scale' were included in this last group.

In all, $63(40.6 \%)$ respondents felt CE checks were a departmental responsibility, $48(31.0 \%)$ felt it to be the doctor's responsibility, and 44 (28.4\%) felt CE checks should be performed by the nursing staff.

\section{Discussion}

IOP measurement is a fundamental part of routine ophthalmic examination and an important variable in the management of glaucoma. ${ }^{2-4}$ The early manifest glaucoma trial showed that decreasing IOP by $1 \mathrm{mmHg}$ leads to a $10 \%$ reduction in the risk of progressive nerve damage. ${ }^{4}$ Documented sources of error in IOP measurement using an applanation tonometer include corneal thickness, eyelid squeezing, and tight neck-ties which overestimate IOP; increased fluorescein and tear-film volume, poor illumination and number of tonometer contacts which underestimate IOP, while corneal astigmatism, interobserver, and CEs have a variable effect on IOP. ${ }^{2,5}$

The manufacturer suggests an acceptable CE of $\pm 0.5 \mathrm{mmHg}$. Tonometers showing a CE beyond this range must be sent for recalibration and removed from clinical practice. ${ }^{1}$ Sandhu et $a l^{2}$ demonstrated that only $10 \%$ of their studied tonometers fell within the manufacturer's CE range. Wessels and $\mathrm{Oh}^{6}$ have demonstrated that tonometers in routine clinical use may develop CE outside the manufacturer's recommendations within two weeks of routine clinical use. They also suggest that new tonometers checked within 3 months of purchase may develop CE in the range of $1.5-3.25 \mathrm{mmHg}^{6}$ Whitacre and $\mathrm{Stein}^{5}$ found an average CE of $\pm 2.0 \mathrm{mmHg}$ in tonometers studied in their clinical environment.

CEs are more likely to deviate into the positive, rather than negative range. This may have the effect of overestimating IOP measurements. This loss of tonometer accuracy may have a significant effect on a patient's clinical management. ${ }^{2,3,6}$

Studies demonstrate that the Goldmann applanation tonometer requires frequent $\mathrm{CE}$ checks to ensure that its accuracy is maintained within the manufacturer's guidelines. $^{2-6}$ To the best of our knowledge, there are no published guidelines regarding the frequency at which CE checks should be made.

Our previous study on the practice of doctors in the UK with regard to CE checks and their opinion on who was responsible for $\mathrm{CE}$ checks showed that $85 \%$ had never checked tonometers for CE. A total of $70 \%$ of responding doctors felt that calibration checks were not their responsibility. They believe that either nursing staff or other hospital staff (eg Medical Physics Department) should carry out calibration checks and ensure the accuracy of tonometers. ${ }^{3}$

Our current survey shows that many eye units are not having $\mathrm{CE}$ checks done on a routine basis. A third have $\mathrm{CE}$ checks done at more than trimonthly intervals, and only a quarter had CE checks done within monthly intervals.

Ideally, tonometers should be checked for CE at each time they are used. Our previous study suggests that despite using a different tonometer by a different doctor for every clinical session, only a minority of units are checking the tonometer for CE on a daily basis. ${ }^{3}$

This survey highlights the lack of consensus regarding who is responsible for CE checks. Despite more than a quarter of the responding nurses believing nurses are responsible for $\mathrm{CE}$ checks, this is not translated into clinical practice. $\mathrm{CE}$ checks are not being performed at a 
sufficient frequency, to ensure the accuracy of tonometers to be maintained. A third of responding nurses believe that it is the doctors who should be responsible for $\mathrm{CE}$ checks and $40 \%$ believe it is a departmental responsibility (eg Medical Engineering).

When taken in context with our previous survey of doctors these findings suggest that the lack of understanding and consensus between the two groups of health-care professionals with regard to $\mathrm{CE}$ checks is leading each side to a feeling that $\mathrm{CE}$ checks are not their responsibility. This results in inadequate $\mathrm{CE}$ checks which may render the instrument outside of its recommended accuracy range.

It is important that all eye units should develop departmental protocols regarding calibration checks. An individual or a group of individuals in each department (nurses/doctors/medical engineers/other trained personnel) must be clearly identified and held accountable for checking the calibration of all departmental tonometers at regular intervals. We recommend that tonometers should be checked for CEs at least on a monthly basis.

\section{Acknowledgements}

Financial or proprietary interest: None.

\section{References}

1 Haag-Streit. Goldmann Applanation Tonometer Instruction Manual. 2003 Available at: www.haag-streit.com. Accessed on 15 May 2007.

2 Sandhu SS, Chattopadhyay S, Birch MK, Ray-Chaudhuri N. Frequency of Goldmann applanation tonometer calibration error checks. J Glaucoma 2005; 14(3): 215-218.

3 Kumar N, Jivan S. Goldmann applanation tonometer calibration error checks: current practice in the UK. Eye 2007; 21: 733-734.

4 Heijl A, Leske M, Bensson B, Hyman L, Bengtsson B, Hussein $\mathrm{M}$ et al. Reduction of intraocular pressure and glaucoma progression: results from the early manifest glaucoma trial. Arch Ophthalmol 2004; 120: 1268-1279.

5 Whitacre M, Stein R. Sources of error with use of Goldmanntype tonometers. Surv Ophthalmol 1993; 38: 1-30.

6 Wessels I, Oh Y. Tonometer utilisation, accuracy, and calibration under field conditions. Arch Ophthalmol 1990; 108: 1709-1712. 